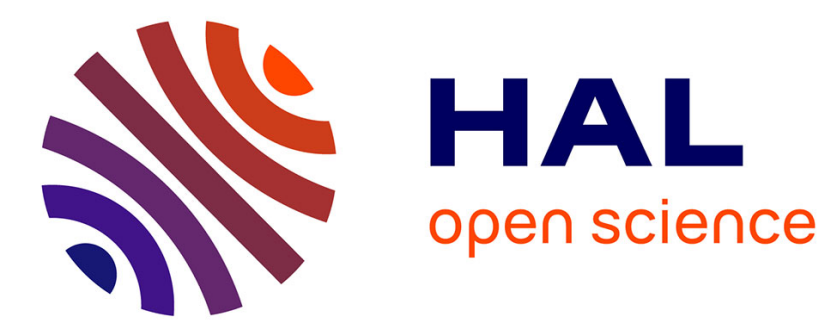

\title{
Contribution à l'étude des propriétés macroscopiques des ferrofluides
}

\author{
J. Barral, Raymonde Bonnefille, M. Duret, M. Kant
}

\section{To cite this version:}

J. Barral, Raymonde Bonnefille, M. Duret, M. Kant. Contribution à l'étude des propriétés macroscopiques des ferrofluides. Revue de Physique Appliquée, 1977, 12 (10), pp.1711-1717. 10.1051/rphysap:0197700120100171100 . jpa-00244391

\section{HAL Id: jpa-00244391 https://hal.science/jpa-00244391}

Submitted on 1 Jan 1977

HAL is a multi-disciplinary open access archive for the deposit and dissemination of scientific research documents, whether they are published or not. The documents may come from teaching and research institutions in France or abroad, or from public or private research centers.
L'archive ouverte pluridisciplinaire HAL, est destinée au dépôt et à la diffusion de documents scientifiques de niveau recherche, publiés ou non, émanant des établissements d'enseignement et de recherche français ou étrangers, des laboratoires publics ou privés. 
Classification

Physics Abstracts

$75.50 \mathrm{M}-75.60 \mathrm{~J}-75.80$

\title{
CONTRIBUTION A L'ÉTUDE DES PROPRIÉTÉS MACROSCOPIQUES DES FERROFLUIDES
}

\author{
J. BARRAL, R. BONNEFILLE, M. DURET et M. KANT \\ Laboratoire d'Électricité Industrielle du Conservatoire National des Arts et Métiers \\ et Laboratoire de Génie Électrique des Universités de Paris VI et Paris XI (*) \\ 33, avenue du Général .Leclerc, 92260 Fontenay-aux-Roses, France
}

(Reçu le 7 avril 1977, accepté le 8 juillet 1977)

\begin{abstract}
Résumé. - On étudie d'abord les propriétés mécaniques et magnétiques, ainsi que les liaisons entre ces propriétés, de trois ferrofluides en vue de leur utilisation dans des convertisseurs électromécaniques d'énergie. On précise ensuite les résultats obtenus lors de l'application, aux ferrofluides considérés, de contraintes simulées telles que le vieillissement, les chocs thermiques et les vibrations.
\end{abstract}

\begin{abstract}
First this article deals with the main mechanical and magnetic properties of the ferrofluids, and the relations between them, with a view to their possible utilization in electromechanical convertors. The results of measurements made after periods under simulated pratical stresses, i.e. ageing. thermal cycles and vibrations. are then given
\end{abstract}

1. Introduction. - En cherchant un moyen simple de transmettre directement des forces à un liquide par l'intermédiaire de champ magnétique, Papell et Faber [1], [2], ont observé qu'ils pouvaient réaliser dans l'entrefer d'un électro-aimant, une suspension de magnétite subdomaniale stabilisée par l'acide oléique.

On connaissait depuis longtemps des suspensions colloïdales de matériaux magnétiques, mais c'était la première fois que ce type de suspensions était considéré comme un continuum liquide apparent à réponse magnétique. Quoique l'existence d'un liquide ferromagnétique homogène soit théoriquement possible, elle n'a donné lieu à aucune réalisation pratique en dépit de nombreuses tentatives effectuées.

Aussi, ces suspensions colloïdales représentent-elles la seule classe de liquides au sein desquels on peut introduire des forces magnétiques d'une certaine importance donnant naissance à un mouvement du fluide. Neuringer et Rosensweig, qui ont été parmi les principaux pionniers en ce domaine, ont donné à ces colloïdes le nom de ferrofluides, qui suggère la combinaison d'une réponse magnétique et de l'état liquide [3], [4], [5].

2. Matériaux étudiés et domaine d'étude. - Nous avons étudié trois ferrofluides désignés ci-après par

$\left(^{*}\right)$ Associé au C.N.R.S les références $200 \mathrm{G}, 400 \mathrm{G}$ et $600 \mathrm{G}$, relatives à leur induction nominale en gauss. Il s'agit de fluides disponibles dans le commerce.

D'après les observations faites au microscope électronique, la taille moyenne des particules est de $100 \AA$; il vaut mieux parler de taille et non de diamètre car si, pour simplifier les calculs, on assimile les petites particules à des sphères, leur forme est, dans la réalité, très différente.

Un fluide, tel que celui référencé $200 \mathrm{G}$, contient environ $10^{17}$ particules par centimètre cube.

On suppose que chaque particule, quels que soient sa taille ou son état de subdivision, conserve la propriété magnétique essentielle d'un domaine.

Nous rappelons que le matériau aggloméré est composé d'un assemblage de domaines dans chacun desquels le moment magnétique par unité de volume est la valeur de saturation appropriée à la température considérée. Le rayon d'un domaine sphérique est de l'ordre de $150 \AA$ pour les matériaux magnétiques ordinaires. Il s'ensuit que les particules contenues dans les solutions étudiées ont généralement la taille d'un sous-domaine

Les ferrofluides $200 \mathrm{G}$ et $400 \mathrm{G}$ ont pour liquide support un hydrocarbure. Le ferrofluide $600 \mathrm{G}$ a un liquide support qui est un ester.

Nous nous limiterons à l'étude des propriétés macroscopiques de ces ferrofluides en vue de leur application ultérieure à des dispositifs de conversion électromécanique d'énergie.

Après la mise en forme de l'équation d'écoulement 
d'un ferrofluide, nous décrirons les mesures concernant trois domaines principaux :

- les propriétés mécaniques,

- les propriétés magnétiques,

- les liaisons entre les propriétés mécaniques et magnétiques.

Nous analyserons ensuite un certain nombre d'essais destinés à simuler les contraintes qui pourraient affecter un ferrofluide dans un convertisseur électromécanique et qui sont essentiellement :

- le vieillissement,

- les chocs thermiques,

- les vibrations.

3. Équation d'écoulement. - En désignant respectivement par $f_{i}$ et $v_{i}$ les composantes $i^{\text {emes }}$ de la force volumique $\mathbf{f}$ et de la vitesse $\boldsymbol{v}$, par $\rho$ la masse volumique, et par $\sigma_{i j}$ la composante $i j$ du tenseur des contraintes $\overline{\bar{\sigma}}$, l'équation régissant l'écoulement d'un fluide s'écrit, avec la convention de sommation sur les indices d'Einstein [6]

$$
f_{i}+\frac{\partial \sigma_{i j}}{\partial x_{j}}=\frac{\mathrm{d} v_{i}}{\mathrm{~d} t}
$$

Le tenseur $\overline{\bar{\sigma}}$ peut se décomposer en une partie symétrique de composante $\sigma_{(i j)}$ et une partie antisymétrique de composante $\sigma_{[i j]}$. Le théorème du moment cinétique permet d'écrire

$$
\sigma_{[12]}=\frac{1}{2} c_{3} ; \quad \sigma_{[23]}=\frac{1}{2} c_{1} ; \quad \sigma_{[31]}=\frac{1}{2} c_{2}
$$

où $c_{k}$ est la $k^{\mathrm{eme}}$ composante du couple volumique $c$. Dans le cas où il n'y a pas d'effet électromagnétique, $c$ est nul et le tenseur des contraintes est, comme il se doit, symétrique.

Pour un fluide incompressible et newtonien, c'est-àdire dont la viscosité cinématique est indépendante $\mathrm{du}$ gradient de vitesse, il est classique de supposer que la partie symétrique de $\sigma_{i j}$ est de la forme

$$
\sigma_{(i j)}=-p \delta_{i j}+\eta\left(\frac{\partial v_{i}}{\partial x_{j}}+\frac{\partial v_{j}}{\partial x_{i}}\right)
$$

où $\delta_{i j}$ est le symbole de Kronecker, $p$ la pression au point considéré et $\eta$ la viscosité dynamique. Cette dernière est une fonction des variables d'état, c'est-àdire, à priori, des variables $p, \rho, T$ (température absolue), $\mathbf{B}$ (induction magnétique) et $\mathbf{M}$ (aimantation).

L'aimantation $M$ dépend, de façon générale, de $\mathbf{B}$, de $T$ et de $\rho$. La masse volumique $\rho$ d'un fluide incompressible n'étant fonction que de $T$, la viscosité dynamique ne dépend donc que de $T$ et de $\mathbf{B}$.

Nous allons montrer qu'un ferrofluide satisfait bien à la loi de comportement (3) [12].

4. Propriétés mécaniques des ferrofluides [7]. Mesure de la viscosité CINÉMATIQUE. - Deux types de viscosimètres à capillaire ont été utilisés :

- le viscosimètre Ubbelohde,

- le viscosimètre Cannon-Fenske.
Dans les deux cas, on mesure le temps d'écoulement $\Delta t$ du liquide dans le capillaire; à une température donnée, la viscosité cinématique $v$ est liée à $\Delta t$ par la relation

$$
\Delta t=K v
$$

où $K$ est une constante caractéristique de l'appareil.

En effectuant plusieurs mesures à différentes valeurs du rayon du capillaire, donc de $K$, c'est-à-dire à différentes vitesses maximales d'écoulement, on constate que $\frac{K}{\Delta t}$ est constant pour une température donnée. Ainsi, la viscosité cinématique $v$ ne dépend pas de la vitesse de déformation : le ferrofluide a bien un comportement newtonien.

La mesure de la masse volumique $\rho$ permet de calculer alors la viscosité dynamique

\begin{tabular}{|c|c|c|c|}
\hline \multicolumn{4}{|c|}{ TABLEAU I } \\
\hline Type de ferrofluide & $200 \mathrm{G}$ & $400 \mathrm{G}$ & $600 \mathrm{G}$ \\
\hline- & - & - & - \\
\hline $\begin{array}{l}\text { Viscosité cinématique } v \\
\left(\mathrm{~m}^{2} / \mathrm{s}\right)\end{array}$ & $4 \times 10^{-6}$ & $5,7 \times 10^{-6}$ & $17,2 \times 10^{-6}$ \\
\hline $\begin{array}{l}\text { Masse volumique } \rho \\
\left(\mathrm{kg} / \mathrm{m}^{3}\right)\end{array}$ & $0,98 \times 10^{3}$ & $1,21 \times 10^{3}$ & $1,45 \times 10^{3}$ \\
\hline $\begin{array}{l}\text { Viscosité dynamique } \\
\quad P 1\end{array}$ & $3.9 \times 10^{-3}$ & $6.9 \times 10^{-3}$ & $25 \times 10^{-3}$ \\
\hline
\end{tabular}

$$
\eta=\rho v
$$

Les divers résultats, obtenus à la température $\theta=25^{\circ} \mathrm{C}$, sont rassemblés dans le tableau ci-dessous :

Les courbes représentant les variations de $v$ en fonction de la température sont tracées. sur la figure 1.

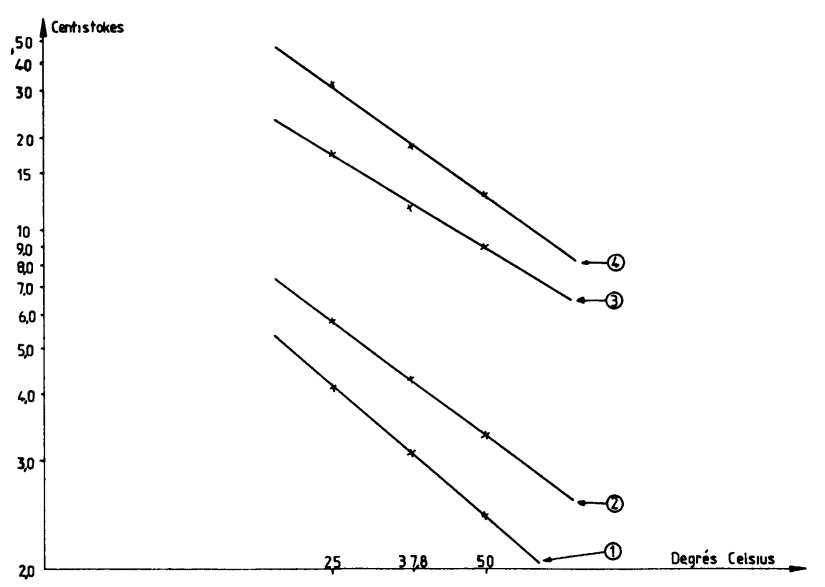

FIG. 1. - Courbes de la viscosité cinématique en fonction de la température.

1 - fluide $200 \mathrm{G}$
2 - fluide $400 \mathrm{G}$
3 - fluide $600 \mathrm{G}$

4 - fluide $600 \mathrm{G}$ (après chocs thermiques).

[Kinematic viscosity curves as a function of temperature.

1 - fluid $200 \mathrm{G}$
2 - fluid $400 \mathrm{G}$
3 - fluid $600 \mathrm{G}$

4 - fluid $600 \mathrm{G}$ (after thermal shocks).] 
5. Propriétés magnétiques des ferrofluides [7], [8]. - 5.1. ÉTABlisSEMENT DE LA COURBE DE MAGNÉTISME B (H) A TEMPÉRATURE DONNÉE. - Pour relever les courbes de magnétisme, on a utilisé un tube en verre en forme de tore (Fig. 2), sur lequel ont été
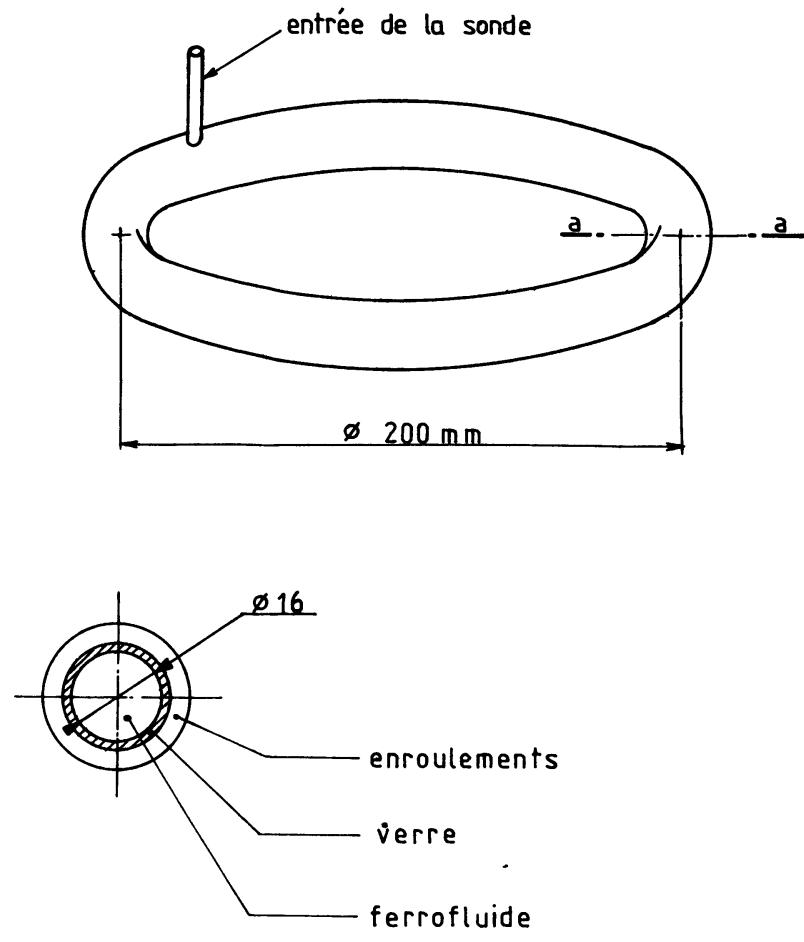

coupe aa

Fig. 2. - Schéma du tore utilisé pour l'établissement des courbes de magnétisme.

[Diagram of the torus used for the determination of magnetic characteristics.]

bobinés un enroulement primaire et un enroulement secondaire. Le tore est enfermé dans une étuve, ce qui permet d'obtenir, pour tout le ferrofluide, une température uniforme. Pour tracer la courbe $B(H)$, on a employé successivement :

- un fluxmètre,

- un gaussmètre et une sonde à effet Hall immergée dans le ferrofluide (le gaussmètre possédant une sortie, les courbes de magnétisme pouvaient être tracées directement sur une table traçante),

- la sonde précédente alimentée avec un courant constant, la tension de Hall étant lue directement sur la table traçante.

Nous nous limiterons à la description de la dernière méthode qui a donné, au vu des essais, les résultats les plus précis (Fig. 3).

La sonde à effet Hall est branchée directement sur une alimentation stabilisée à courant constant. Cette sonde, étant très plate et positionnée perpendiculairement aux lignes de champ dans le tore, mesure effectivement l'induction régnant dans le ferrofluide. Au-dessus de la sonde est fixée la soudure chaude

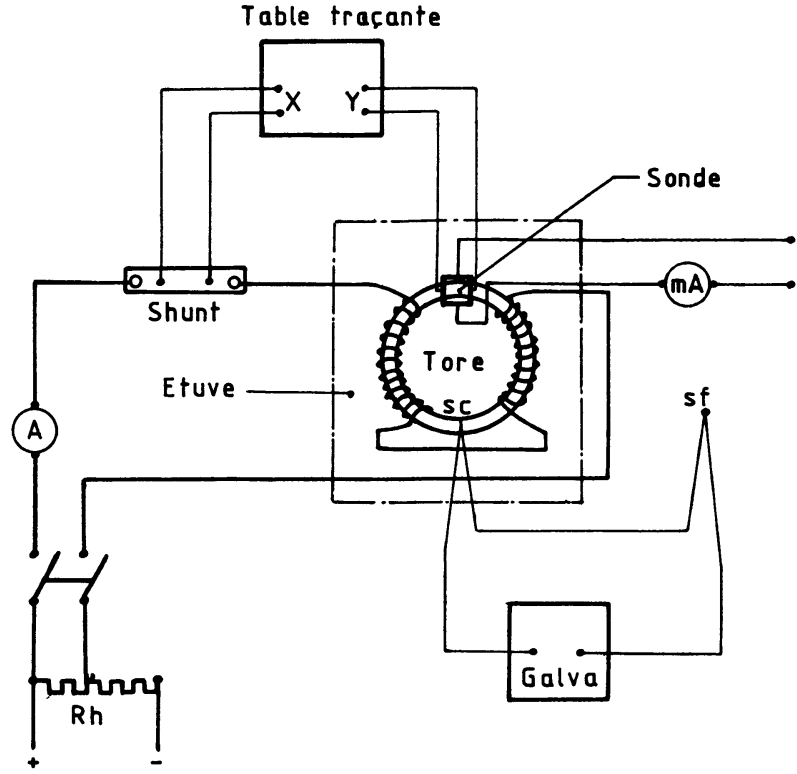

Fig. 3. - Tracé des courbes de magnétisme avec variation de la température.

[Plotting of magnetic curves with variation of temperature.]

d'un thermocouple (cette position supérieure a été choisie pour éviter l'action des fils métalliques sur la mesure de l'induction), dont la soudure froide se trouve dans la glace fondante. Après étalonnage, on peut donc lire directement, sur un galvanomètre, la température du ferrofluide. Les résultats obtenus se traduisent par des courbes de $B$ en fonction de $H$ tracées sur la table pour différentes températures du ferrofluide.

Pour avoir les résultats les plus justes, on reprend les valeurs des courbes tracées et on applique la correction due à la température de la sonde.

Les figures 4,5 et 6 représentent les courbes de $B$ en fonction de $H$ pour les ferrofluides $200 \mathrm{G}, 400 \mathrm{G}$ et $600 \mathrm{G}$.

Ces courbes mettent en évidence l'absence de

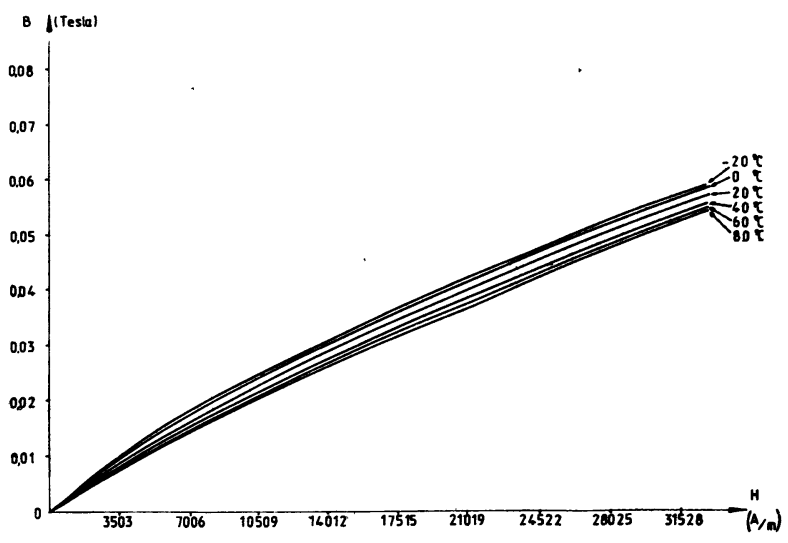

FIG. 4. - Courbes de magnétisme (fluide $200 \mathrm{G}$ ).

[Magnetization curves (fluid $200 \mathrm{G}$ ).] 


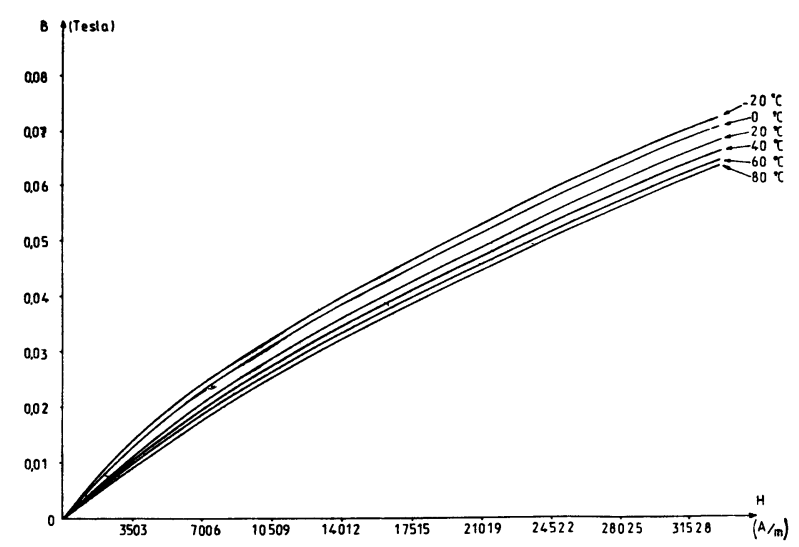

Fig. 5. - Courbes de magnétisme (fluide $400 \mathrm{G}$ ).

[Magnetization curves (fluid $400 \mathrm{G}$ ).]

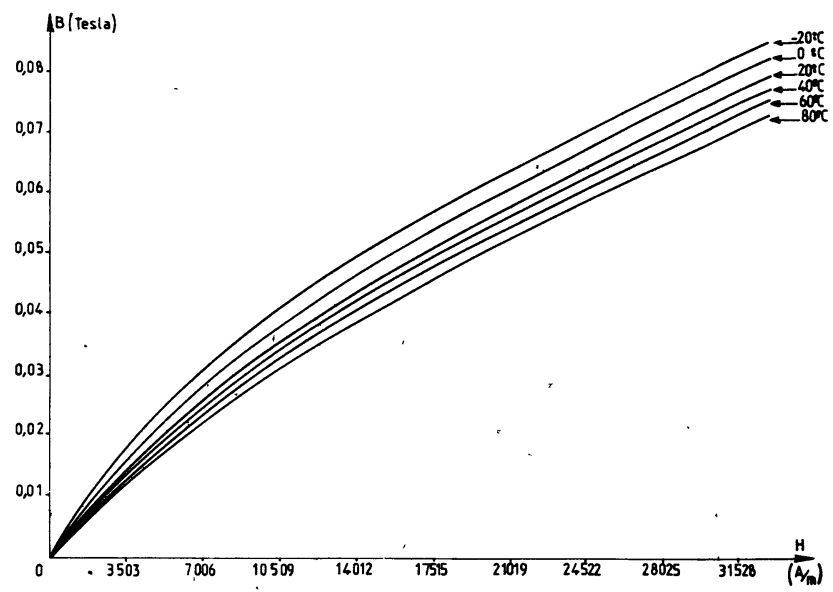

FIG. 6. - Courbes de magnétisme (fluide $600 \mathrm{G}$ ).

[Magnetization curves (fluid $600 \mathrm{G}$ ).]

phénomène d'hystérésis; une explication possible est que, les particules étant de taille subdomaniale et se trouvant dans un liquide support, les rotations d'aimantation dans les domaines s'opèrent de manière continue et pratiquement instantannée, ce qui annule l'hystérésis.

Sur la figure 7, sont tracées les courbes donnant, en fonction de $H$, le produit $\chi T\left(\chi=\frac{M}{H}\right.$, susceptibilité magnétique) pour les trois types de ferrofluides.

L'allure de ces courbes et le fait que la perméabilité relative initiale $\mu_{\mathrm{r}}$ des ferrofluides est comprise entre 1 et 2 (voir les courbes 4,5 et 6 ) confirment les propriétés superparamagnétiques de ces liquides.

Ces différentes expériences (comme le test de la table vibrante le confirmera plus nettement par la suite) montrent que le ferrofluide se comporte, au point de vue magnétique, comme un milieu isotrope. Ce résultat, qui était prévisible, compte tenu de la forme quasi sphérique des particules et de l'homo-

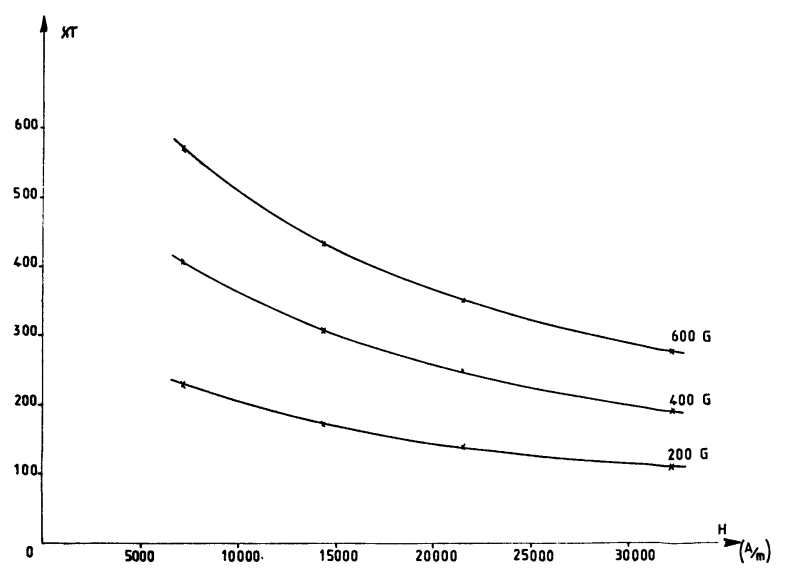

FIG. 7. - Courbes $\chi T(H)$.

[Curves $\chi T(H)$.]

généité du mélange, se traduit mathématiquement par la colinéarité des vecteurs $H$ et $B$, en chaque point du liquide. Ce fait a d'ailleurs été vérifié lors de l'expérience suivante : après avoir positionné la sonde dans le ferrofluide de manière à ce qu'elle soit perpendiculaire au vecteur champ magnétique produit par la bobine, on a fait tourner la sonde autour de son axe jusqu'à ce qu'elle soit parallèle à ce vecteur; on s'aperçoit alors que le flux de B est maximal quand la sonde est dans le plan de la bobine (Fig. 8) et nul quand elle est perpendiculaire au plan de la bobine (Fig. 9), les vecteurs $\mathbf{B}$ et $\mathbf{H}$ sont donc bien colinéaires et proportionnels.

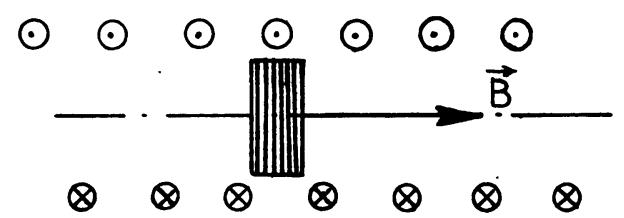

Fig. 8. - Flux maximal (sonde dans le plan de la bobine).

[Maximal flux position (probe in the coil plane).]
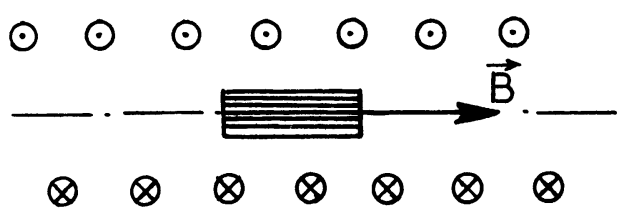

Fig. 9. - Flux nul (sonde perpendiculaire au plan de la bobine). [Zero flux position (probe perpendicular to the coil plane).]

5.2. DÉTERMINATION THÉORIQUE DE LA PERMÉABILITÉ RELATIVE. - Le ferrofluide étant un mélange à dispersion fine, dont les particules sont assimilables à des sphères de diamètre moyen égal à quelques 
$100 \AA$, on peut considérer l'induction et le champ magnétique moyens dans de grands volumes (quelques $\mathrm{mm}^{3}$ ) par rapport aux particules. A l'égard de tels champs moyens, le mélange est un milieu homogène et isotrope et peut être caractérisé par une certaine valeur $\mu_{\mathrm{r}}$ de la perméabilité relative.

En supposant la température constante, un calcul classique [9] montre que, en désignant par $\mu_{\mathrm{rm}}$ la perméabilité relative et par $C_{\mathrm{m}}=\frac{V_{\mathrm{m}}}{V}$, la concentration en volume de la matière magnétique, on a

$$
\mu_{\mathrm{r}}=1+3 C_{\mathrm{m}} \frac{\mu_{\mathrm{rm}}-1}{\mu_{\mathrm{rm}}+2}
$$

La perméabilité relative de la magnétite non saturée variant de 10 à 1000 , on peut donc écrire :

$$
\mu_{\mathrm{r}} \approx 1+3 C_{\mathrm{m}}
$$

Prenons 2 exemples, en considérant des particules de diamètre moyen égal à $100 \AA$.

a) Pour un ferrofluide contenant $10^{17}$ particules magnétiques par centimètre cube, il vient

$$
C_{\mathrm{m}}=\frac{V_{\mathrm{m}}}{V}=\frac{4}{3} \pi \quad \times \quad \frac{\left(10^{-6}\right)^{3}}{8} \times 10^{17} \approx 0,05
$$

d'où $\mu_{\mathrm{r}}=1,15$.

b) Pour un ferrofluide contenant $10^{18}$ particules par $\mathrm{cm}^{3}$, il vient

$$
C_{\mathrm{m}}=\frac{V_{\mathrm{m}}}{V}=\frac{4}{3} \pi \quad \times \quad \frac{\left(10^{-6}\right)^{3}}{8} \times 10^{18} \approx 0,5
$$

d'où $\mu_{\mathrm{r}}=2,5$.

On retrouve les ordres de grandeur donnés par l'expérience; il est important de remarquer que $\mu_{\mathrm{r}}$ aura toujours une valeur inférieure à 4 (du moins tant que les particules sont assimilables à des sphères).

6. Liaisons entre les propriétés mécaniques et magnétiques des ferrofluides. - Nous avons étudié la viscosité d'un ferrofluide placé dans un écoulement soumis à un champ d'induction magnétique radial.

6.1. ÉTUDE THÉORIQUE. - La figure 10 représente, en coupe, deux cylindres coaxiaux, de rayons respectifs $R_{1}$ et $R_{2}$ et de hauteur $h$, entre lesquels peut circuler un ferrofluide, mis en mouvement par un cylindre creux de rayon $R_{3}$, tournant à la vitesse angulaire $\mathbf{\Omega}$.

Le liquide étant isotrope au point de vue magnétique, il n'y a pas de couple volumique et le tenseur des contraintes est symétrique, soit, d'après (3)

$$
\sigma_{i j}=\sigma_{(i j)}=-p \delta_{i j}+\eta\left(\frac{\partial v_{i}}{\partial x_{j}}+\frac{\partial v_{j}}{\partial x_{i}}\right)
$$

L'équation d'écoulement (1) s'écrit alors :

$$
\begin{aligned}
\rho \frac{\mathrm{d} v}{\mathrm{~d} t}=\rho \frac{\partial v}{\partial t}+\rho(\boldsymbol{v} \nabla) & v= \\
= & -\nabla p+\eta \dot{\nabla}^{2} \mathbf{v}+\rho \mathbf{g}+\mathbf{f}_{\mathrm{m}}
\end{aligned}
$$

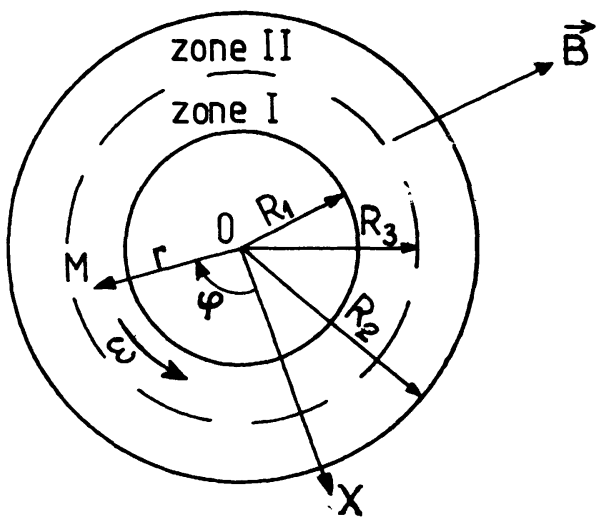

Fig. 10. - Mesure de la viscosité dynamique dans le champ radial.

[Measurement of dynamic viscosity in radial field.;

où $\mathbf{f}_{\mathbf{m}}$ est la force magnétique volumique

$$
\mathbf{f}_{\mathrm{m}}=(\mathbf{M} \nabla) \mathbf{B}=\frac{\left(\mu_{\mathrm{r}}-1\right)}{\mu_{\mathrm{o}} \mu_{\mathrm{r}}}(\mathbf{B} \nabla) \mathbf{B}
$$

Le champ appliqué étant radial, les composantes de $\mathbf{f}_{\mathrm{m}}$ dans le système de coordonnées cylindriques $(\varphi, r)$ (Fig. 10) sont :

$$
\begin{aligned}
& \left(\mathbf{f}_{\mathrm{m}}\right)_{r}=\frac{\left(\mu_{\mathrm{r}}-1\right)}{\mu_{\mathrm{o}} \mu_{\mathrm{r}}} B_{r} \cdot \frac{\partial B_{r}}{\partial r} \\
& \left(\mathbf{f}_{\mathrm{m}}\right)_{\varphi}=0
\end{aligned}
$$

L'écoulement étant supposé stationnaire, on peut poser :

$$
\rho \frac{\partial v}{\partial t}=0
$$

Par raisón de symétrie, $v_{r}=0$, alors que $v_{\varphi}$ et $p$ ne dépendent que de $r$. De (5) on déduit alors :

$$
\left[\nabla^{2} \dot{\mathbf{v}}\right]_{\varphi}=\frac{\partial^{2} v_{\varphi}}{\partial r^{2}}+\frac{1}{r} \frac{\partial v_{\varphi}}{\partial r}-\frac{v_{\varphi}}{r^{2}}=0
$$

La solution de (6) est de la forme :

$$
v_{\varphi}=a r+\frac{b}{r}
$$

Des conditions aux limites

$$
v_{\varphi}\left(R_{1}\right)=v_{\varphi}\left(R_{2}\right)=0 ; \quad v_{\varphi}\left(R_{3}\right)=\Omega R_{3}
$$

on tire la váleur des constantes $a$ et $b$ dans les deux zones I et II.

On peut alors calculer le moment $m$ des forces de frottement agissant sur les cylindres extérieurs :

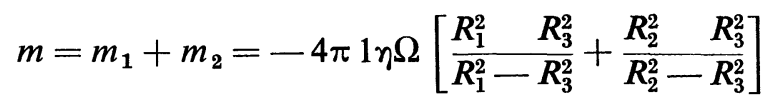

On constate que le moment $m$ ne dépend, pour une vitesse donnée, que de la viscosité dynamique $\eta$. 
Une variation éventuelle de $m$ avec $B$ met donc en évidence un effet de magnéto-viscosité.

6.2. ÉTUDE EXPÉRIMENTALE. - La réalisation pratique de l'étude ci-dessus a été effectuée à l'aide d'un viscosimètre rotatif. Une sonde à effet Hall, branchée directement sur un gaussmètre, sert à la mesure de $B$ dans l'entrefer; un thermocouple étalonné permet de s'assurer de la constance de la température durant la mesure; elle est en fait toujours restée comprise entre $25^{\circ} \mathrm{C}$ et $26^{\circ} \mathrm{C}$. Les courbes $\eta(B)$ obtenues sont tracées sur la figure 11 .

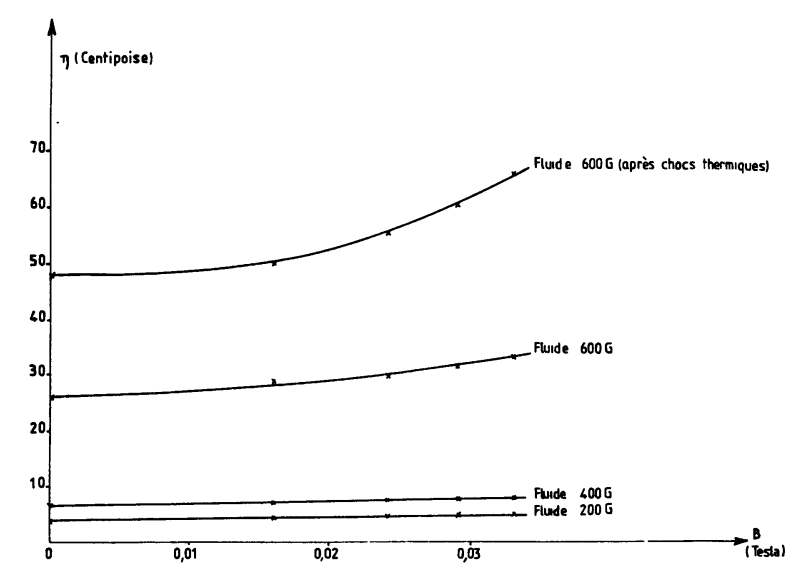

FIG. 11. - Courbes de la viscosité dynamique en fonction de l'induction.

[Dynamic viscosity curves as a function of induction.]

6.3. INTERPRÉTATION. - a) On constate, en premier lieu, qu'il n'y a aucune aimantation des petites particules de magnétite car, après avoir tracé chaque courbe, un essai à $B=0$ a été fait et la nouvelle mesure était identique à la mesure initiale.

b) Les essais confirment bien que la viscosité est indépendante de la vitesse du ferrofluide. En effet, pour une même induction et différentes vitesses, on a trouvé la même viscosité dynamique. Ceci confirme encore que le ferrofluide a un comportement newtonien (les vitesses ont été choisies pour avoir, dans ce dernier cas, un écoulement laminaire).

c) On peut interpréter la force de viscosité comme étant la contrainte de cisaillement s'exerçant entre deux filets fluides de vitesses très peu différentes. Cette contrainte sera d'autant plus forte que les particules entre les filets seront plus liées. Or, en présence du champ, les particules de magnétite s'aimantent et, par suite, s'attirent mutuellement.

On peut en déduire que la contrainte de cisaillement sera plus forte. Tout se passe comme si $\eta$ augmentait avec $B$. On peut écrire :

$$
\eta=\eta_{0}+\eta_{1} B+\eta_{2} B^{2}+\ldots
$$

Pour un ferrofluide donné, si $B$ est faible, le terme $\mathrm{du} 1^{\mathrm{er}}$ ordre suffit; puis, quand $B$ augmente, l'influence du terme en $B^{2}$ devient importante. La loi de $\eta$ en fonction de $B$ est parabolique pour les valeurs moyennes de l'induction; plus la viscosité est forte, plus la parabole s'incurve rapidement, ce qui apparaît sur les courbes de la figure 11.

7. Essais de contraintes. - 7.1. ESSAIS DE VIEILLISSEMENT. - Ces mesures ont permis de préciser le comportement du ferrofluide dans le temps. On a tracé, quatre mois après les premières mesures et à des températures variant de $-20^{\circ} \mathrm{C}$ à $+80^{\circ} \mathrm{C}$, les courbes de magnétisme $B(H)$ qui se sont révélées confondues avec celles des figures 4,5 et 6 .

On peut en conclure que les ferrofluides étudiés ont des propriétés stables dans le temps. Les forces de cohésion qui permettent aux particules de rester en suspension semblent donc indépendantes du temps. Après avoir transvasé du ferrofluide, resté 8 mois dans une bouteille fermée, nous n'avons constaté aucun dépôt, ce qui confirme bien les résultats précédents.

7.2. ESSAIS AUX CHOCS THERMIQUES. - Après avoir maintenu pendant plusieurs minutes le ferrofluide en état d'ébullition $\left(\theta \approx 190^{\circ} \mathrm{C}\right)$, on le ramène à sa température initiale $\left(\theta \approx 20^{\circ} \mathrm{C}\right)$ et on relève la caractéristique $B(H)$.

Les essais ont été effectués avec le ferrofluide $600 \mathrm{G}$ et les courbes $B(H)$, tracées avec l'appareillage représenté sur la figure 3 , sont portées sur la figure 12 .

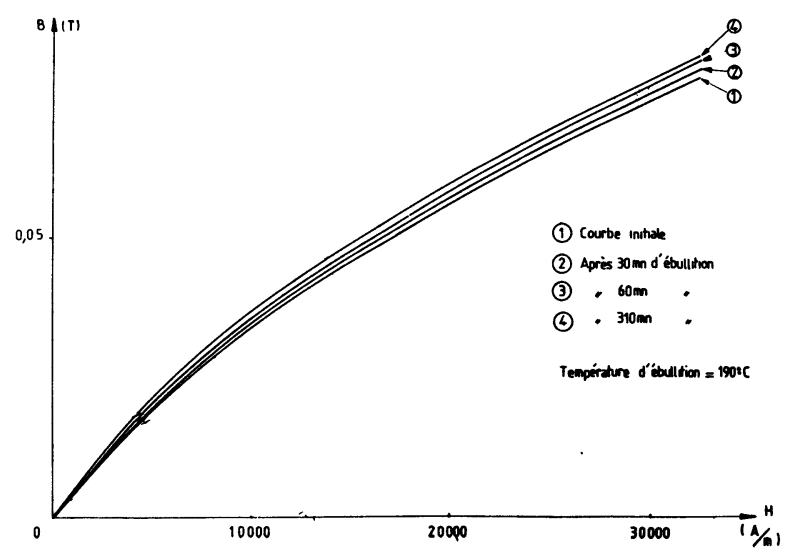

FIG. 12. - Courbes d'aimantation après chocs thermiques (température: $20^{\circ} \mathrm{C}$ ).

[Magnetization curves after thermal shocks (temperature : $20^{\circ} \mathrm{C}$ ). ]

Ces courbes montrent que, pour un champ magnétique donné, l'induction augmente avec le nombre de chocs thermiques. Nous pensons que ce phénomène est dû au fait que, malgré les précautions prises, il y a une évaporation du liquide support pendant les essais et, partant, augmentation de la concentration $C_{\mathrm{m}}$, ce qui correspond, d'après (4), à un accroissement de la perméabilité relative $\mu_{\mathrm{r}}$.

On a également constaté à l'aide, d'un aimant, 
placé à côté du ballon de verre contenant le ferrofluide, que ce dernier conserve ses propriétés magnétiques, même au voisinage de sa température d'ébullition.

7.3. ESSAIS SUR TABLE VIBRANTE. - On a disposé l'appareillage représenté sur la figure 3 sur une table vibrante dont on peut régler l'amplitude $(0,2 \mathrm{~mm}$ à $2 \mathrm{~mm})$ et la fréquence $(0 \mathrm{à} 80 \mathrm{~Hz})$ des vibrations. Les mesures, faites à une seule température $\left(\theta=40^{\circ} \mathrm{C}\right)$, n'ont révélé aucune différence appréciable entre les nombreuses courbes $B(H)$ relevées à diverses fréquences et amplitudes des vibrations, ce qui montre que ces dernières n'ont pas d'influence sur les propriétés magnétiques des ferrofluides.

8. Conclusions. - Des études et des mesures ci-dessus développées, on peut tirer les conclusions suivantes :

- La viscosité cinématique, mesurée en l'absence du champ magnétique et en fonction de la température étant indépendante du gradient de vitesse, on en déduit que le ferrofluide est un fluide newtonien dans la limite des températures étudiées.

- Le tracé des courbes de magnétisme, à différentes températures, et sur la table vibrante, montre qu'il n'existe pas d'hystérésis, que le ferrofluide est isotrope et homogène et qu'il a un comportement superparamagnétique.
- La loi de variation de la viscosité dynamique d'un ferrofluide, en mouvement dans un champ d'induction magnétique perpendiculaire au sens de l'écoulement, est, pour des valeurs modestes de l'induction, parabolique. De plus, en comparant avec des résultats obtenus par ailleurs [10] on constate que la variation de viscosité du ferrofluide dans un champ perpendiculaire est égale à la moitié de celle qui est obtenue dans un champ parallèle à l'écoulement.

Remarquons que, dans les limites d'induction et de vitesse étudiées, les résultats obtenus semblent indiquer que l'on peut admettre le comportement simplifié du ferrofluide que nous proposons, sans avoir recours à des modèles plus élaborés [11]; probablement plus conformes à la réalité. Enfin les ferrofluides considérés sont stables dans le temps, ils conservent leurs propriétés magnétiques jusqu'à leur température d'ébullition et indépendamment des vibrations qui leur sont appliquées.

Remerciements. - Les auteurs remercient M. Elnekavé, chef de Service au Laboratoire Central des Industries Électriques et M. Martinet, Maître de Recherche au Laboratoire de Physique des Solides de l'Université de Paris Sud pour leur contribution à la mise en œuvre des dispositifs de mesure utilisés.

\section{Bibliographie}

1. Articles auxquels se rapportent directement les travaux ci-dessus.

[1] PAPell S. S. and FABER O. C., Zero and reduced-gravity simulation on a magnetic-colloid poolboiling system, NASA Tech. Note (1965) 3288.

[2] PApell S. S. and Faber O. C., on the influence of non uniform magnetic fields on ferromagnetics colloidal sols. NASA Tech. Note (1968) 4676.

[3] Neuringer J. L. and Rosensweig R. E., ferrohydrodynamics Phys. Fluids, 7. Number 12 (1964).

[4] Rosensweig R. E., fluidmagnetic buoyency. A.I.A.A., 4. Number 10 (1966).

[5] Rosensweig R. E., ferrohydrodynamics. Encyclopedic Dictionnary of Physics. 14 (1971).

[6] Germain P., mécanique des milieux continus (MASSON), tome I, 1973, p. 197.

\section{Autres articles interessants.}

BERTRAND A.R.V., les ferrofluides. Rev. IFP. XXV, $\mathrm{n}^{\circ} 1$ (1970).

HALL W. F. and BUSENBERG N., viscosity of magnetic suspensions. J. Chem. Phys. 51. Number 1 (1969).

MAILFERT R. and MARTINET A., flow regimes for a magnetic suspension under a rotating magnetic field. J. de Physique $\mathbf{3 4}$ (1973).
[7] DURET M., contributions à l'étude des ferrofluides. Mémoire d'Ingénieur CNAM (1976).

[8] KAISER R. and MisKolcZY G., magnétic properties of stable dispersions of subdomain magnetite particles. J. Appl. Phys. 41. Number 3 (1970).

[9] LANDAU L., LIFCHITZ E., electrodynamique des milieux continus. (Éditions MIR. Moscou) 1971.

[10] TAGUE MC., J.P. magnetoviscosity of magnetic colloids. $J$. Chem. Phys. 51. Number 1. (1969).

[11] Jenkins J. T., some simple flows of a para-magnetic fluid. J. Physique 32 (1971) 931.

[12] TARAPOV I. E., the hydromatics of polarizing and magnetizing media Magnetohydrodynamics. 8, No. 1 (1974).

Moskowitz R. and Rosensweig R. E., non-me chanical torquedriven flow of a ferromagnetic fluid by an electromagnetic field. Appl. Phys. Lett. 11. Number 10 (1967).

Martinet A., biréfringence et dichroïsme linéaire des ferrofluides sous champ magnétique. Rheol. Acta. 13, nº 2 (1974). 